A novel fabrication process for out-of-plane microneedle sheets of biocompatible polymer

This article has been downloaded from IOPscience. Please scroll down to see the full text article.

2007 J. Micromech. Microeng. 171184

(http://iopscience.iop.org/0960-1317/17/6/012)

View the table of contents for this issue, or go to the journal homepage for more

Download details:

IP Address: 143.248.233.201

The article was downloaded on 13/04/2011 at 08:30

Please note that terms and conditions apply. 


\title{
A novel fabrication process for out-of-plane microneedle sheets of biocompatible polymer
}

\author{
Manhee Han ${ }^{1}$, Dong-Hun Hyun ${ }^{2}$, Hyoun-Hyang Park ${ }^{1}$, \\ Seung S Lee ${ }^{1}$, Chang-Hyeon Kim ${ }^{2}$ and ChangGyou Kim ${ }^{2}$ \\ ${ }^{1}$ Department of Mechanical Engineering and Center for Ultramicrochemical Process \\ Systems, Korea Advanced Institute of Science and Technology, 373-1 Guseong-dong, \\ Yuseong-gu, Daejeon, Korea \\ ${ }^{2}$ Daedeok Research Institute, Honam Petrochemical Corporation, 24-1 Jang-dong, \\ Yuseong-gu, Daejeon, Korea \\ E-mail: manhee@kaist.ac.kr
}

Received 8 November 2006, in final form 27 February 2007

Published 15 May 2007

Online at stacks.iop.org/JMM/17/1184

\begin{abstract}
This paper presents a novel process for fabricating out-of-plane microneedle sheets of biocompatible polymer using in-plane microneedles. This process comprises four steps: (1) fabrication of in-plane microneedles using inclined UV lithography and electroforming, (2) conversion of the in-plane microneedles to an out-of-plane microneedle array, (3) fabrication of a negative PDMS mold and (4) fabrication of out-of-plane microneedle sheets of biocompatible polymer by hot embossing. The in-plane microneedles are fabricated with a sharp tip for low insertion forces and are made long to ensure sufficient penetration depth. The in-plane microneedles are converted into an out-of-plane microneedle array to increase the needle density. The negative mold is fabricated for mass-production using a polymer molding technique. The final out-of-plane microneedle sheets are produced using polycarbonate for biocompatibility by employing the hot embossing process. The height of the fabricated needles ranges from 500 to $1500 \mu \mathrm{m}$, and the distance between the needles is 500 to $2000 \mu \mathrm{m}$. The radii of curvature are approximately $2 \mu \mathrm{m}$, while the tip angles are in the range of $39-56^{\circ}$. Most of the geometrical characteristics of the out-of-plane microneedles can be freely controlled for real life applications such as drug delivery, cosmetic delivery and mesotherapy. Since it is also possible to mass-produce the microneedles, this novel process holds sufficient potential for applications in industrial fields.
\end{abstract}

(Some figures in this article are in colour only in the electronic version)

\section{Introduction}

The delivery of drugs into the body through the oral route is problematic mainly due to the degradation of drugs in the gastrointestinal tract and their elimination through the liver. Further, the delivery of drugs using conventional hypodermic needles is painful for patients and is not appropriate for long-term, continuous deliveries [1]. An alternative delivery technique employs a transdermal patch. However, transdermal delivery is severely limited by the inability of a large majority of drugs to enter the body through the skin at therapeutic rates because the outer layer of the skin (stratum corneum), which is approximately 10-20 $\mu \mathrm{m}$ thick and composed of keratinized dead cells and scales, acts as a barrier [2].

Therefore, in order to increase skin permeability the stratum corneum has to be disrupted. Several approaches have been studied to disrupt this layer; they range from chemical/lipid enhancers [3,4] to electric fields employing 
iontophoresis and electroporation $[5,6]$ to pressure waves generated by ultrasound or photoacoustic effects $[7,8]$. These enhancements have only had a limited impact on medical practices to date because chemical methods can affect the skin or the drug, while physical methods require complex systems.

Microneedles, which are effective in forming micro perforations in the stratum corneum, are one of the few promising minimally invasive drug delivery devices [9]. They do not cause chemical effects on the skin or the drug and do not require an energy source or other complex systems. Microneedles are classified as in-plane and out-of-plane microneedles. The longitudinal axes of the former are parallel to the substrate and those of the latter are perpendicular to the substrate. In the case of in-plane microneedles, although it is easy to control the length, side shape and tip sharpness, the drug delivery rate is low due to the low density of the needles and small volume of the microchannels inside the needles $[10,11]$. On the other hand, in the case of out-of-plane microneedles, although it is difficult to control the length, side shape and tip sharpness, the drug delivery rate is high due to the high density of the needles [9, 12-15].

In addition, in most of the previous studies, microneedles have been made of silicon or metal; therefore, they could either be incompatible with a biological tissue or could leave residues after their removal from the tissue [16]. In recent studies, microneedles have been fabricated using polymers [1, 17-20]. Polymers are not only inexpensive and mass-producible but some of them are also biocompatible and biodegradable. However, the previously used polymer microneedles also possess the same limitation as that mentioned for the out-ofplane microneedles [17-19], or they may involve complicated fabrication processes and low accessibility to $\mathrm{x}$-ray sources $[1,20]$.

This paper presents a novel process for fabricating outof-plane microneedle sheets of biocompatible polymer using in-plane microneedles in order to overcome the limitations of existing microneedles. The proposed process comprises four steps: (1) fabrication of in-plane microneedles, (2) conversion of the in-plane microneedles to an out-of-plane microneedle array, (3) fabrication of a negative mold and (4) fabrication of out-of-plane microneedle sheets of biocompatible polymer.

\section{Fabrication concept and process design}

Figure 1 outlines the steps to fabricate polymer out-ofplane microneedle sheets. First, in-plane microneedles are fabricated (figure 1(a)). In this step, it is easy to control the length, side shape and tip sharpness. Next, the in-plane microneedles are converted to an out-of-plane microneedle array to increase the needle density (figure $1(b)$ ). For mass production, a negative mold is fabricated by replicating the out-of-plane microneedle array in figure 1(c). Finally, outof-plane microneedle sheets of biocompatible polymer are fabricated by using the negative mold in a hot embossing machine (figure $1(d)$ ).

\subsection{In-plane microneedle}

In-plane microneedles are fabricated by using inclined UV lithography and nickel-electroforming, as illustrated in

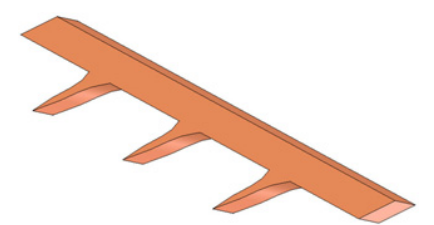

(a)

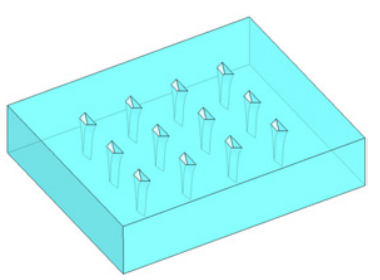

(c)

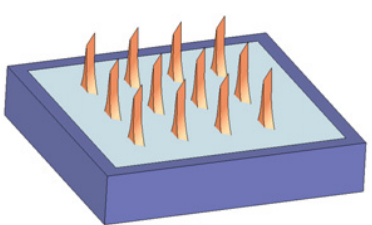

(b)

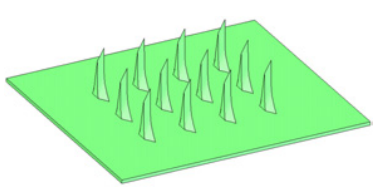

$(d)$
Figure 1. Steps to fabricate polymer out-of-plane microneedle sheets: (a) fabrication of in-plane microneedles using inclined UV lithography and electroforming, $(b)$ conversion of the in-plane microneedles to an out-of-plane microneedle array, $(c)$ fabrication of a negative PDMS mold and $(d)$ fabrication of polymer out-of-plane microneedle sheets by hot embossing.

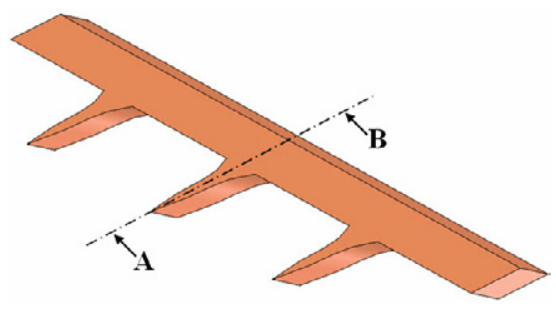

(a)
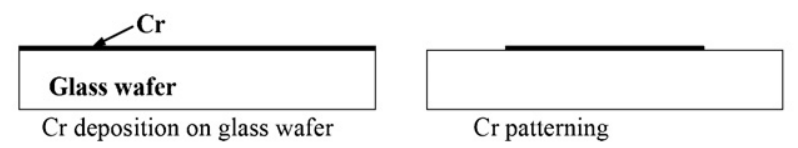

$\mathrm{Cr}$ deposition on glass wafer
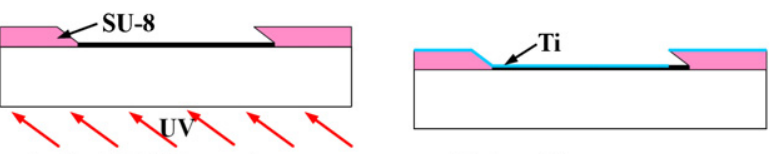

Inclined UV lithography

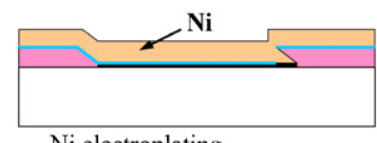

Ni electroplating

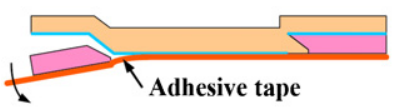

Ti deposition

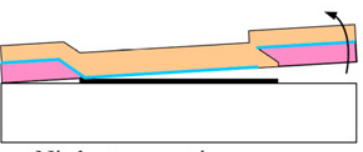

Ni sheet separation

SU-8 stripping and Ti removal

Chemical-mechanical polishing

(b)

Figure 2. Process for fabricating in-plane microneedles: (a) schematic view of the in-plane microneedles and $(b)$ fabrication process along the cross-section along lines A-B shown in $(a)$.

figure 2. First, a $150 \mathrm{~nm}$ thick chromium (Cr) layer is deposited on to a Pyrex glass wafer (Corning). After the $\mathrm{Cr}$ layer is patterned to define the shape of the microneedle, an SU-8 structure is formed on the Cr-patterned glass wafer by inclined 


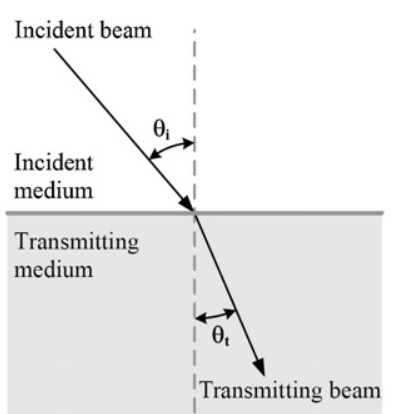

(a)

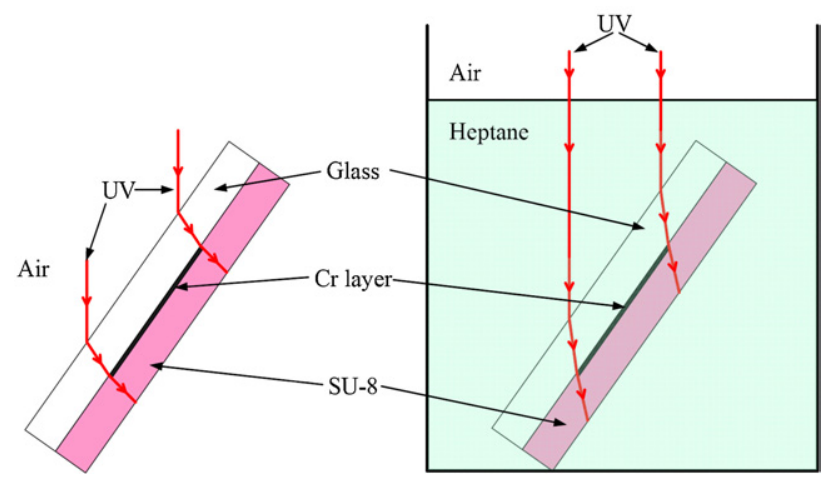

(b)

Figure 3. Propagation routes of the UV radiation in the inclined exposure process: $(a)$ refraction of the light beam at the interface between two different transparent media; $(b)$ incident medium: air; (c) incident medium: heptane.

UV lithography [21-23]. In this step, the SU-8 layer should be thicker than the desired height of the in-plane microneedle. The UV irradiation is performed from the backside of the glass wafer so that the Cr layer acts as a UV mask. If the in-plane microneedles can be obtained directly using SU-8, this process would be much faster and would include fewer processing steps. However, SU-8 microneedles are too brittle and fragile.

In order to obtain metal microneedles which are easy to handle, a titanium (Ti) layer is deposited as a seed layer for electroplating, and a $\mathrm{Ni}$ layer is formed on the entire upper surface of the wafer by electroplating. Subsequently, the $\mathrm{Ni}$ sheet is stripped off the wafer by hand. In this step, the boundaries between the Ni layer and the wafer and between the SU-8 layer and the wafer separate out. The SU-8 layer embedded in the $\mathrm{Ni}$ sheet is stripped using an adhesive tape. The Ti layer on the Ni layer is removed by dipping the sheet in hydrofluoric acid. To obtain the final in-plane microneedles, the Ni sheet is polished to the desired thickness of the microneedles using chemical-mechanical polishing.

When conducting inclined UV exposure, the route of UV radiation must be considered. This is because the $\mathrm{UV}$ radiation is refracted as it enters the SU-8 layer, as shown in figure 3 . The relationship between the incident angle $\left(\theta_{i}\right)$ and the refraction angle $\left(\theta_{t}\right)$ is determined by the law of refraction (Snell's law) as follows:

$$
n_{i} \sin \theta_{i}=n_{t} \sin \theta_{t}
$$

where $n_{i}$ and $n_{t}$ are the refractive indices of the incident and transmitting media, respectively.
According to this law, the refraction angle is determined by the incident angle and the refractive index of the surrounding medium without considering the influence of the refractive index of the glass substrate as follows, if total reflection has not occurred:

$$
\begin{aligned}
\theta_{\mathrm{SU}-8} & =\sin ^{-1}\left(\frac{n_{\text {glass }}}{n_{\text {SU-8 }}} \sin \theta_{\text {glass }}\right) \\
& =\sin ^{-1}\left(\frac{n_{\text {glass }}}{n_{\text {SU-8 }}} \frac{n_{\text {sur }}}{n_{\text {glass }}} \sin \theta_{\text {sur }}\right) \\
& =\sin ^{-1}\left(\frac{n_{\text {sur }}}{n_{\text {SU-8 }}} \sin \theta_{\text {sur }}\right) .
\end{aligned}
$$

In this study, total reflection has not occurred at any angle because $n_{\text {sur }}\left(n_{\text {air }}=1\right.$ or $\left.n_{\text {heptane }}=1.39\right)<n_{\text {Pyrex }}=1.47$ (Coring) $<n_{\text {SU-8 }}=1.67$ (MicroChem Corp.).

When the surrounding medium is air $\left(n_{\text {air }}=1\right)$ and the incident angle of the UV radiation is $70^{\circ}$, the refraction angle is calculated at $34^{\circ}$, i.e., the angle of the microneedle tip is expected to be $56^{\circ}$. Further, the minimum angle of the microneedle tip is approximately $53^{\circ}$ because the refractive index of SU-8 is approximately 1.67 [24]. To increase the sharpness of the microneedles, a medium with a refractive index greater than $n=1$ should be used as the surrounding medium [25]. Heptane has been selected as the surrounding medium in this study because it does not affect SU-8. Moreover, it is a commonly available material, and can be easily removed by blowing. When an SU-8-coated substrate is placed in heptane and the incident angle of the UV radiation is $70^{\circ}$, the angle of the microneedle tip is expected to be $39^{\circ}$.

\subsection{Out-of-plane microneedle}

The out-of-plane microneedle array is fabricated by arranging the Ni in-plane microneedles perpendicular to the substrate, as shown in figure 4 . In this process, the rectangular spacers not only create spaces between the neighboring in-plane microneedles but also maintain these in-plane microneedles perpendicular to the flat of the jig; this is because the spacers and microneedles are tightly fixed to each other with screws. Finally, polydimethylsiloxane (PDMS; Sylgard 184, Dow Corning) fills the gaps among the microneedles, spacers and a jig, and covers the spacers slightly (the usage of PDMS is mentioned in the following paragragh).

The negative mold illustrated in figure $1(c)$ is fabricated by replicating the out-of-plane microneedle array using a PDMS replication technique [17-19,26]. The PDMS prepolymer and curing agent are mixed in a 10:1 weight ratio and degassed. This mixture is then poured onto the out-of-plane microneedle array in figure $4(b)$, and cured for $12 \mathrm{~h}$ at $40{ }^{\circ} \mathrm{C}$ in an oven. The PDMS replica is then separated from the array. This replica is used as the negative mold for producing the out-of-plane microneedle sheets of polymer. In this step, the PDMS layer for the mold does not stick to the first PDMS layer covering the Ni microneedle and spacer layers. Thus, the Ni needle array can be re-used.

As a microneedle has a higher aspect ratio, hot embossing is most suitable among all the other micro-molding processes for thermoplastic polymers [27]. Figure 5 shows a custommade hot embossing machine used in this study. The upper and 


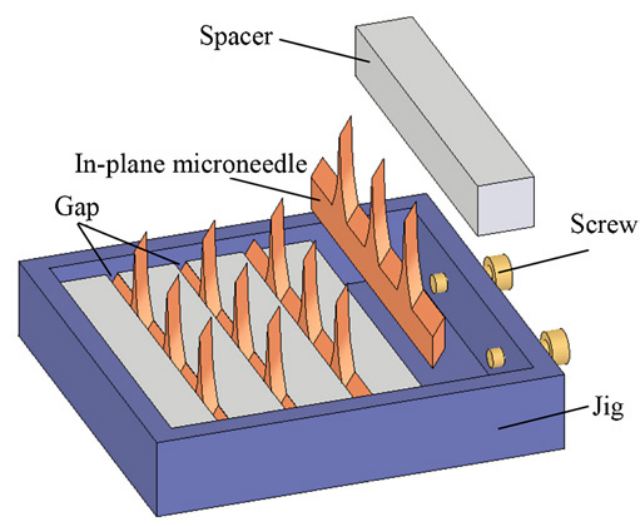

(a)

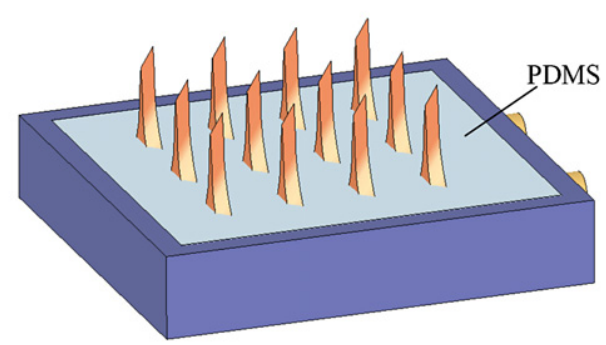

(b)

Figure 4. Conversion of the in-plane microneedles to an out-of-plane microneedle array: $(a)$ arrangement of the in-plane microneedles and spacers and $(b)$ filling of the gaps with PDMS.

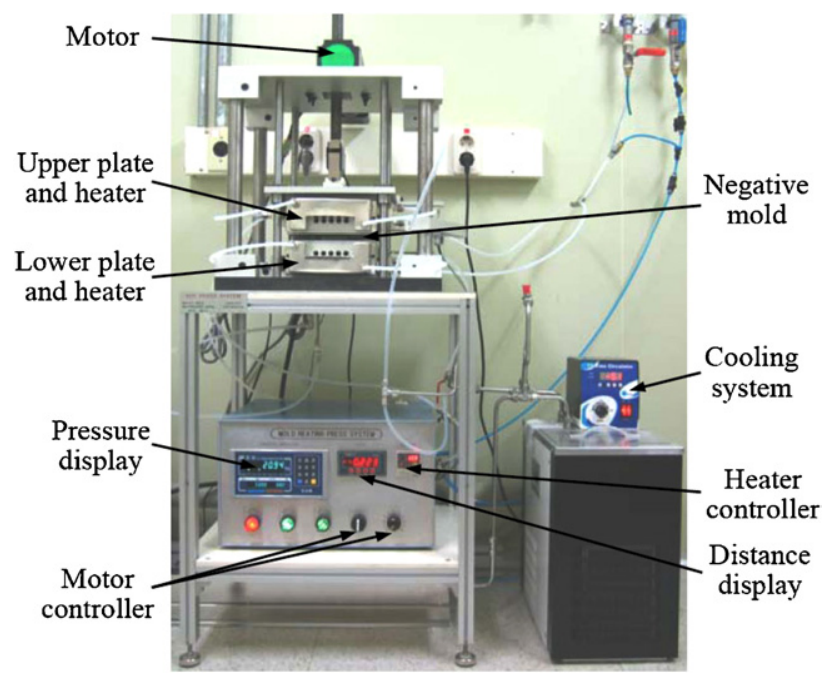

Figure 5. Hot embossing machine.

lower plates of the machine are heated using electric heaters, pressurized using a motor, and cooled using a cooling system. The maximum load is $2 \mathrm{kN}$, the maximum temperature is $350{ }^{\circ} \mathrm{C}$ and the maximum size of a mold insert is $3 \mathrm{~cm} \times$ $3 \mathrm{~cm}$.

Figure 6 shows the hot embossing process for fabricating the out-of-plane microneedle sheets of biocompatible polycarbonate (PC) by using the hot embossing machine. First, biocompatible $\mathrm{PC}$ grains are placed on the negative mold (size: $3 \mathrm{~cm} \times 3 \mathrm{~cm}$ ), which in turn is placed on a Teflon sheet. For

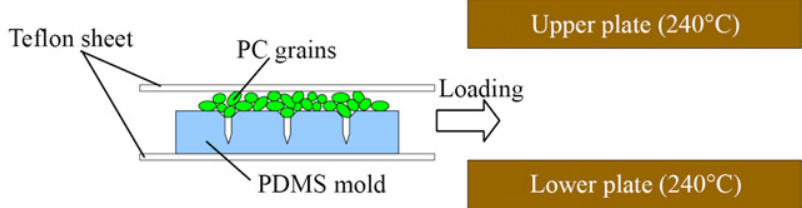

(a)

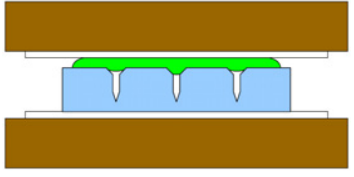

(b)

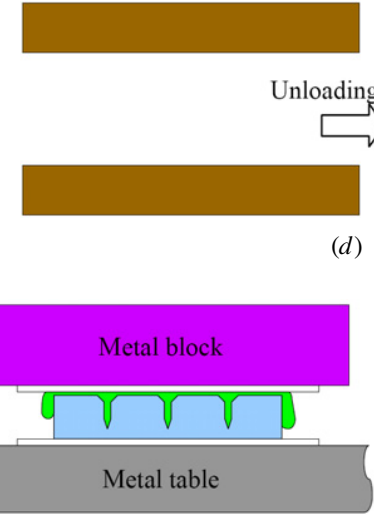

$(e)$

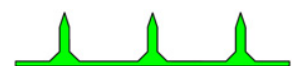

$(f)$
Figure 6. Fabrication of biocompatible PC out-of-plane microneedle sheets: $(a)$ loading the stack comprising a PDMS mold, PC grains and Teflon sheets on to the hot embossing machine,

$(b)$ heating, $(c)$ pressing, $(d)$ unloading the stack from the machine, (e) pressing and cooling and $(f)$ demolding and cutting.

easier handling, a PC plate can be used instead of PC grains. Another Teflon sheet is placed over the PC grains, and the stack is inserted between the upper and lower plates; the temperature of each plate is $240{ }^{\circ} \mathrm{C}$. This stack is heated for $8 \mathrm{~min}$ and pressed with a pressure of $10 \mathrm{~kg} \mathrm{~cm}^{-2}$ for $2 \mathrm{~min}$. The stack is then unloaded from the machine in order to cool it rapidly and pressed with a $5 \mathrm{~kg}$ metal block to prevent deformation. After the stack is cooled for $5 \mathrm{~min}$ at room temperature, the PC microneedle sheet is demolded and the excess PC that has overflowed is cut off.

The usage of a PDMS mold has certain advantages; the fabrication process is simpler and the time required for its fabrication is less in comparison to a metal mold. In addition, it is easy to demold the PC microneedle sheet from the PDMS mold even though there are no ejection holes and pins. Although the endurance of the PDMS mold is less than that of a metal mold, the PDMS mold can be re-used many times. In fact, the mold used in this study remained intact in spite of being used hundreds of times.

In the hot embossing technique for the fabrication of microneedles, the insertion of the polymer into the mold can be improved by using a vacuum $[18,19,27]$. However, the machine would be complex and the application of a vacuum would increase the process time. In this study, the process temperature is increased up to approximately the melting 


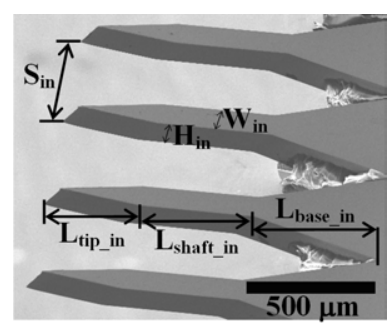

(a)

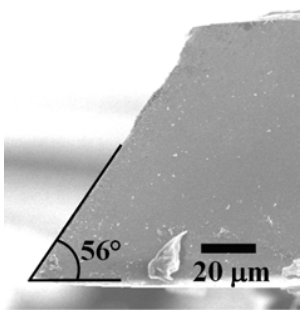

(c)

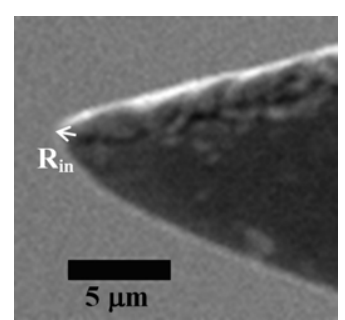

(b)

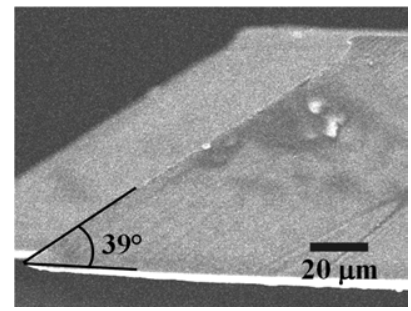

$(d)$
Figure 7. SEM images of the Ni in-plane microneedles when the incident angle of the UV radiation is $70^{\circ}:(a)$ array, $(b)$ tip, $(c)$ side view when the incident medium is air and $(d)$ sideview when the incident medium is heptane.

temperature (melting temperature of $\mathrm{PC}$ is $265^{\circ} \mathrm{C}$ ) instead of applying vacuum at temperatures above the glass transition temperature (glass transition temperature of $\mathrm{PC}$ is $150{ }^{\circ} \mathrm{C}$ ) in order to simplify the machine and reduce the processing time.

\section{Fabrication results}

Figure 7 shows SEM images of the fabricated Ni in-plane microneedles when the incident angle of the UV radiation is approximately $70^{\circ}$ in the exposure process. The radii of the curvature $\left(R_{\text {in }}\right)$ of the in-plane microneedles are less than $1 \mu \mathrm{m}$; the lengths of the tips $\left(L_{\text {tip_in }}\right)$, shafts $\left(L_{\text {shaft_in }}\right)$ and bases $\left(L_{\text {base_in }}\right)$ are $60-120 \mu \mathrm{m}$, approximately $500 \mu \mathrm{m}$ and approximately $600 \mu \mathrm{m}$, respectively. The widths $\left(W_{\text {in }}\right)$, heights $\left(H_{\text {in }}\right)$ and spacing $\left(S_{\text {in }}\right)$ of the needles are $100 \mu \mathrm{m}$, $100 \mu \mathrm{m}$ and $500 \mu \mathrm{m}$, respectively. Most of the geometrical characteristics of an in-plane microneedle can be controlled by adjusting the size, shape and spacing of the lithographic mask, and the process conditions, such as the thickness of the SU-8 layer, the incident angle of the UV radiation and the thickness of the Ni sheet after the polishing process.

When the surrounding medium was air and the incident angle of the UV radiation was approximately $70^{\circ}$, the angle of a $\mathrm{Ni}$ microneedle tip was approximately $56^{\circ}$, as shown in figure 7(c). When an SU-8-coated substrate was placed in heptane and the incident angle of the UV radiation was approximately $70^{\circ}$, the angle of a $\mathrm{Ni}$ microneedle tip was approximately $39^{\circ}$, as shown in figure $7(d)$. The measured angles of the Ni microneedle tip equal its calculated angles of in accordance with Snell's law.

Figure 8 shows images of the PC out-of-plane microneedle sheets. The heights $\left(H_{\text {out }}\right)$ of the out-of-plane microneedles, shown in figures $8(a),(b),(c)$ and $(d)$, are approximately $1.5 \mathrm{~mm}, 1.0 \mathrm{~mm}, 1.0 \mathrm{~mm}$ and $500 \mu \mathrm{m}$, respectively. The heights of the out-of-plane microneedles can be controlled by adjusting the lengths of the in-plane microneedles and the filling level of PDMS. The spacings $\left(S_{\text {out }}\right)$ of the outof-plane microneedles in figures $8(a)-(d)$ are approximately $2.0 \mathrm{~mm}, 1.0 \mathrm{~mm}, 1.0 \mathrm{~mm}$ and $500 \mu \mathrm{m}$, respectively. The spacings of the out-of-plane microneedles can be controlled by changing the widths of the spacers and the spacings of the lithographic mask. The radii $\left(R_{\text {out }}\right)$ of the curvature of the out-of-plane microneedles are larger than those of the in-plane microneedles, but the radii of curvature of the former are still as small as approximately $2 \mu \mathrm{m}$, as shown in figure 8(e).

Another advantage of this process is that the ratio among the lengths of the tips ( $\left.L_{\text {tip_out }}\right)$, shafts $\left(L_{\text {shaft_out }}\right)$ and bases ( $\left.L_{\text {base_out }}\right)$ can be controlled freely. For example, the total lengths of the microneedles in figure $8(c)$ are the same, but the shaft lengths of the microneedles in the front are greater than those in the rear. Thus, it is possible to design an appropriate base that does not penetrate the skin; however, it becomes imperative to overcome certain barriers such as the wrinkles in the skin and the dimpling of the skin during insertion.

In addition, these microneedle sheets are safe for humans as they are made of biocompatible PC. Because this process uses a polymer molding technique, the microneedles can be formed with not only PC but also with other biocompatible polymers such as polylactic acid and polyglycolic acid, which are used by other groups [18].

\section{Penetration test and applications of the PC microneedle sheets}

To observe the penetration of the microneedle sheet into rat skin, experiments were conducted using a bromocresol green (MW 720) solution. A $500 \mu \mathrm{m}$ long microneedle sheet was inserted into rat skin that was gripped by two clips for $1 \mathrm{~min}$. After removing the microneedle sheet, the bromocresol green solution was placed onto the skin, swabbed for $3 \mathrm{~min}$ and then wiped off to stain the sites of needle penetration. Figure 9 shows images from beneath the skin surface; these images indicate that the microneedles penetrated into the skin. Thus, the microneedle sheets can be applied to make holes directly in the skin surface prior to drug delivery applied to the surface. Another way of using this microneedle sheet is by first coating the needles with a drug and then inserting them into the skin [28].

Using this novel fabrication process, most of the geometrical characteristics of PC out-of-plane microneedle sheets, such as the height, spacing, thickness and the ratio among the lengths of the tip, shaft and base can be freely adjusted for varied applications such as cosmetic delivery, drug delivery and mesotherapy (a microinjection technique to reduce or remove fat, wrinkles, scars, etc). For cosmetic delivery, the heights of the microneedles range from 50 to $200 \mu \mathrm{m}$ to ensure safety. For drug delivery, the heights range from 100 to $1000 \mu \mathrm{m}$ for the purpose of efficiency and painlessness. In mesotherapy, the heights range from 300 to $2000 \mu \mathrm{m}$ because inflammation is required even though it is painful. Further, the microneedle sheets are biocompatible and a low insertion force is required since the radii of the tips are of the order of a few micrometers [29]. Thus, the microneedle sheets can be used in various real life applications. 


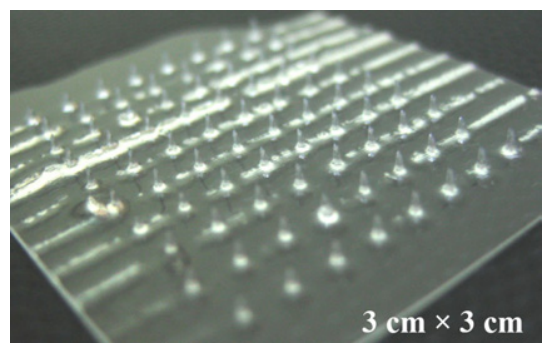

(a)

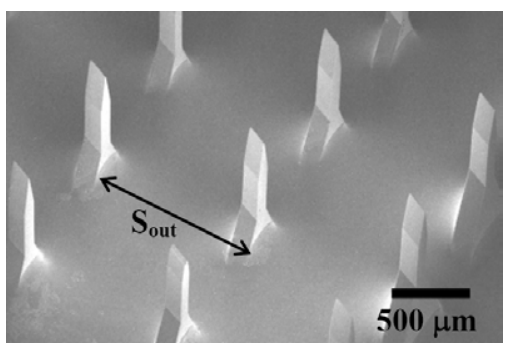

(b)

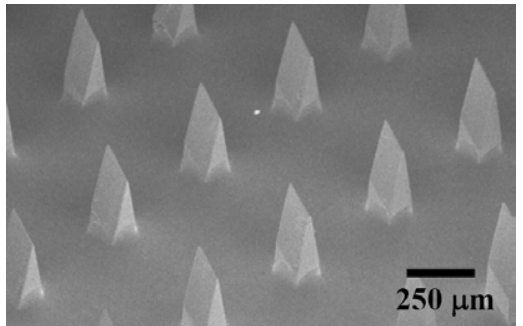

(d)

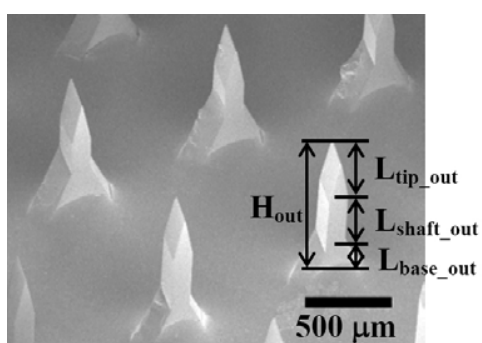

(c)

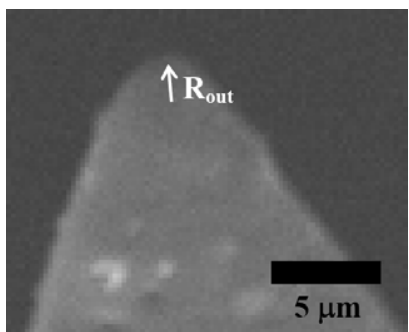

(e)

Figure 8. Images of the PC out-of-plane microneedle sheets produced by the hot embossing process: $(a)$ photograph, $(b)-(d)$ SEM images of the array and $(e)$ a SEM image of the tip.

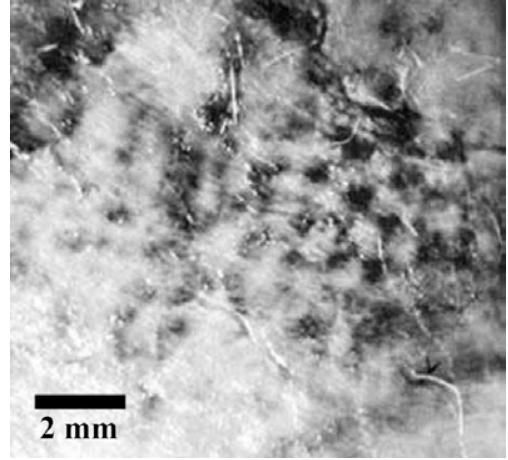

(a)

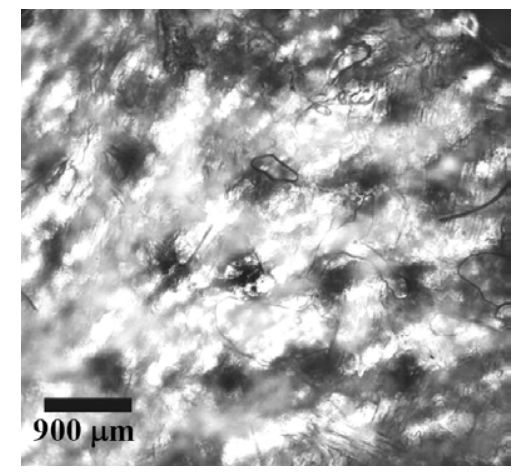

(b)

Figure 9. Skin piercing verification of the microneedle array: $(a)$ image from beneath the rat skin surface in vitro after insertion and removal of the microneedles and $(b)$ photomicrograph of $(a)$.

Figure 10 shows one example of the application of the biocompatible polymer out-of-plane microneedles. The polymer microneedle roller shown in this figure can replace Dermaroller $^{\mathrm{TM}}$ (www.microneedle.com), which consists of stainless steel microneedles, if the polymer microneedles and insertion bars are mass-produced as a single unit using a biocompatible polymer. Then the polymer microneedle rollers would have the advantages of being low-priced and sanitary as compared to existing microneedles.

The fabrication process makes it possible to massproduce cheap microneedle sheets because it uses a polymer molding technique. In addition, the facilities required to fabricate the negative molds of the microneedle sheets comprise only the equipment for UV lithography, metal 


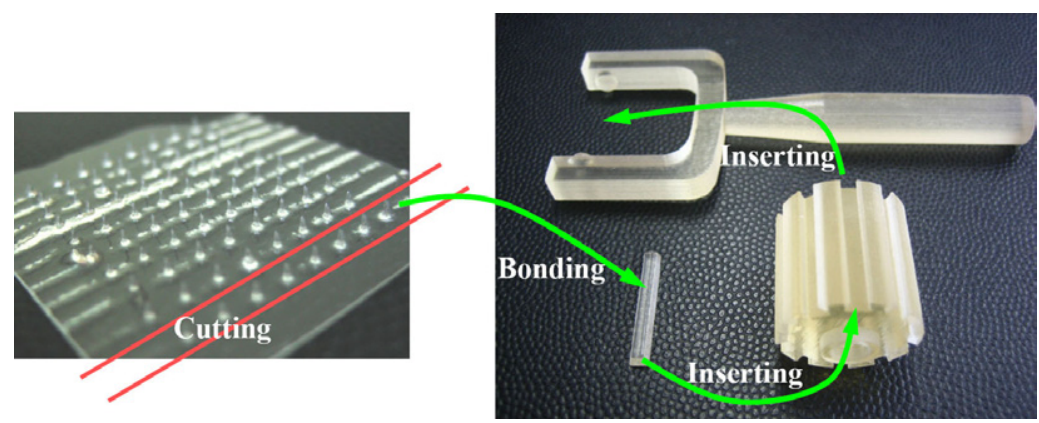

(a)
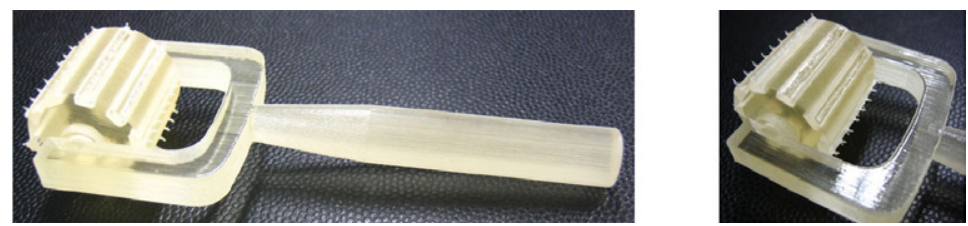

(b)

Figure 10. PC microneedle roller (roller head diameter: $2 \mathrm{~cm}$ ): $(a)$ assembling process and $(b)$ an assembled roller.

deposition, electroplating and polishing. Thus, the process for fabricating the biocompatible PC out-of-plane microneedle sheets holds sufficient potential for applications in industrial fields.

\section{Conclusion}

This study proposes a novel process for fabricating outof-plane microneedle sheets of a biocompatible polymer. The process comprises four steps: (1) fabrication of inplane microneedles using inclined UV lithography and electroforming, (2) conversion of the in-plane microneedles to an out-of-plane microneedle array, (3) fabrication of a negative PDMS mold and (4) fabrication of out-of-plane microneedle sheets of the polymer by hot embossing. The fabricated inplane microneedles have sharp tips and long shafts; thus a low insertion force is required and sufficient penetration depth is achieved. The needle density can be increased by converting the in-plane microneedles to an out-of-plane microneedle array; this results in sufficient drug delivery rates. The hot embossing process using biocompatible polycarbonate enables mass production and provides biocompatibility.

Using this process, most of the geometrical characteristics of a biocompatible out-of-plane microneedle sheet, such as the angle of the tip, the width and height of the shaft, the ratio among the lengths of the tip, shaft and base, and the spacing between the needles, can be controlled freely. Thus, it is possible to fabricate safe microneedles that are suitable for various applications. Furthermore, mass production is possible using polymer molding techniques such as the hot embossing process. Thus, the proposed fabrication method for microneedles is suitable for industrial applications.

\section{Acknowledgments}

This research has been supported by the Intelligent Microsystem Center (IMC; http://www.microsystem.re.kr), which carries out one of the 21st century's Frontier R\&D Projects sponsored by the Korea Ministry of Commerce, Industry and Energy.

\section{References}

[1] Moon S-J and Lee S S 2003 Fabrication of microneedle array using inclined LIGA process Transducers' 03 (Boston, MA) pp 1546-9

[2] Touitou E 2002 Drug delivery across the skin Expert Opin. Biol. Ther 2 723-33

[3] Williams A C and Barry B W 2004 Penetration enhancers Adv. Drug Deliv. Rev. 56 603-18

[4] Cevc G 2004 Lipid vesicles and other colloids as drug carriers on the skin Adv. Drug Deliv. Rev. 56 675-711

[5] Kalia Y N, Naik A, Garrison J and Guy R H 2004 Iontophoretic drug delivery Adv. Drug Deliv. Rev. 56 619-58

[6] Denet A-R, Vanbever R and Preat V 2004 Skin electroporation for transdermaland topical delivery Adv. Drug Deliv. Rev. 56 659-74

[7] Doukas A G and Kollias N 2004 Transdermal delivery with a pressure wave Adv. Drug Deliv. Rev. 56 559-79

[8] Mitragotri S and Kost J 2004 Low-frequency sonophoresis: a review Adv. Drug Deliv. Rev. 56 589-601

[9] Henry S, McAllister D V, Allen M G and Prausnitz M R 1998 Microfabricated microneedles: a novel approach to transdermal drug delivery J. Pharm. Sci. 87 922-5

[10] Reed M L and Lye W-K 2004 Microsystems for drug and gene delivery Proc. IEEE 92 56-75

[11] Zhang P and Jullien G A 2003 Micromachined needles for microbiological sample and drug delivery system ICMENS 2003 (Alberta, Canada, Jul. 20-23) T3A2

[12] Griss P, Tolvanen-Laakso H K, Meriläinen P and Stemme G 2002 Characterization of micromachined spiked biopotential electrodes IEEE Trans. Biomed. Eng. 49 597-604

[13] Griss P and Stemme G 2003 Side-opened out-of-plane microneedles for microfluidic transdermal liquid transfer J. Microelectromech. Syst. 12 296-301

[14] Shikida M, Odagaki M, Todoroki N, Ando M, Ishihara Y, Ando T and Sato K 2004 Non-photolithographic pattern trasfer for fabricating arrayed three-dimensional 
microstructures by chemical anisotropic etching Sensors Actuators A 116 264-71

[15] Trautmann A, Heuck F, Denfeld R, Ruther P and Paul O 2006 Detachable silicon microneedle stamps for allergy skin prick testing Proc. IEEE Conf. MEMS (Istanbul, Turkey) pp 434-7

[16] Stupar P A and Pisano A P 2001 Silicon, parylene, and silicon/parylene micro-needles for strength and toughness Transducers' 01 (Munich, Germany, Jun. 10-14) 4B1.09P

[17] Park J-H, Davis S, Yoon Y-K, Prausnitz M R and Allen M G 2003 Micromachined biodegradable microstructures Proc. IEEE Conf. MEMS (Kyoto, Japan) pp 371-4

[18] Park J-H, Allen M G and Prausnitz M R 2005 Biodegradable polymer microneedles: fabrication, mechanics and transdermal drug delivery J. Control. Release 104 51-66

[19] Trautmann A, Heuck F, Mueller C, Ruther P and Paul O 2005 Replication of microneedle arrays using vacuum casting and hot embossing Transducers' 05 (Seoul, Korea) pp 1420-1423

[20] Moon S-J and Lee S S 2005 A novel fabrication method of a microneedle array using inclined deep x-ray exposure J. Micromech. Microeng. 15 903-11

[21] Han M, Lee W, Lee S-K and Lee S S 2002 Microfabrication of 3D oblique structures by inclined UV lithography Proc. microTAS 2002 vol 1 pp 106-8

[22] Yoon Y-K, Park J-H, Cros F and Allen M G 2003 Integrated vertical screen microfilter system using inclined SU-8
Structures Proc. IEEE Conf. MEMS (Kyoto, Japan) pp 227-30

[23] Sato H, Kakinuma T, Go J S and Shoji S 2003 A novel fabrication of in-channel 3-D micromesh structure using maskless multi-angle exposure and its microfilter application Proc. IEEE Conf. MEMS (Kyoto, Japan) pp 223-6

[24] Han M, Lee W, Lee S-K and Lee S S 2004 3D microfabrication with inclined/rotated UV lithography Sensors Actuators A 111 14-20

[25] Tseng F-G and Hu H-T 2003 A novel micro optical system employing inclined polymer mirrors and fresnel lens for monolithic integration of optical disk pickup heads Transducers' 03 (Boston, MA) pp 599-602

[26] Duffy D C, McDonald J C, Schueller O J A and Whitesides G M 1998 Rapid prototyping of microfluidic systems in poly (dimethylsiloxane) Anal. Chem. 70 4974-84

[27] Heckele M and Schomburg W K 2004 Review on micro molding of thermoplastic polymer J. Micromech. Microeng. 14 R1-R14

[28] Prausnitz M R 2004 Microneedles for transdermal drug delivery Adv. Drug Deliv. Rev. 56 581-7

[29] Davis S P, Landis B J, Adams Z H, Allen M G and Prausnitz M R 2004 Insertion of microneedles into skin: measurement and prediction of insertion force and needle fracture force J. Biomech. 37 1155-63 